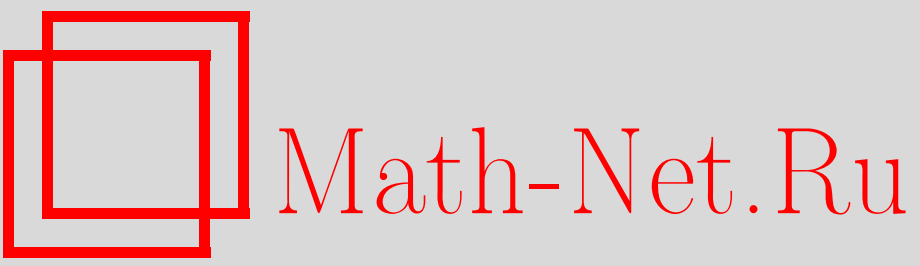

Г. М. Жислин, Спектральные свойства гамильтонианов заряженных систем в однородном магнитном поле. І. Общая характеристика спектра, ТМФ, 2002, том 133, номер 1, 87-102

DOI: https://doi.org/10.4213/tmf382

Использование Общероссийского математического портала Math-Net.Ru подразумевает, что вы прочитали и согласны с пользовательским соглашением

http: //www . mathnet.ru/rus/agreement

Параметры загрузки:

IP : 54.224 .187 .69

26 апреля 2023 г., 17:57:59 
ТЕОРЕТИЧЕСКАЯ

И МАТЕМАТИЧЕСКАЯ

ФИЗИКА

Том 133, № 1

октябрь, 2002

(C) 2002 г.

Г.М. Жислин ${ }^{*}$

\title{
СПЕКТРАЛЬНЫЕ СВОЙСТВА ГАМИЛЬТОНИАНОВ ЗАРЯЖЕННЫХ СИСТЕМ В ОДНОРОДНОМ МАГНИТНОМ ПОЛЕ. І. ОБШАЯ ХАРАКТЕРИСТИКА СПЕКТРА
}

\begin{abstract}
Исследуется спектр гамильтонианов заряженных многочастичных систем в однородном магнитном поле при фиксации суммы $P_{\Sigma}$ компонент псевдомомента и без нее. Доказывается, что при фиксации $P_{\Sigma}$ спектр гамильтонианов не зависит от величины $P_{\Sigma}$, а спектр без фиксации $P_{\Sigma}$ совпадает со спектром при фиксации, отличаясь от последнего лишь дополнительным бесконечным вырождением (что принципиально отличает задачи с однородным магнитным полем от задач без поля, где отсутствие фиксации полного момента приводит к "замазыванию" спектра относительного движения непрерывным спектром). Находится сплошной спектр гамильтонианов. Дается характеристика спектра гамильтонианов двухкластерных не взаимодействующих друг с другом систем, полученных распадением исходной системы из состояния с фиксированным значением $P_{\Sigma}$. Последний результат необходим для изучения чисто точечного спектра.
\end{abstract}

Ключевые слова: гамильтониан, однородное магнитное поле, спектральные свойства, относительное движение, псевдомомент.

\section{1. ВВЕДЕНИЕ}

В настоящей работе изучаются спектральные свойства гамильтонианов квантовых систем $Z_{1}$, состоящих из $n$ частиц с конечными массами в однородном магнитном поле. Предполагается, что суммарный заряд всех частиц $Q \neq 0$. Нас будет интересовать главным образом спектр относительного движения системы $Z_{1}$. Для получения гамильтониана относительного движения в отсутствие магнитного поля отделяют движение центра масс (ЦМ) в $R^{3}$, однако при наличии поля такое отделение в общем случае возможно лишь в направлении поля, т. е. оси $z$. Для построения гамильтониана с магнитным полем, отвечаюшего относительному движению и в плоскости $x, y$, было предложено [1] рассматривать ограничения оператора, полученного после отделения движения ЦМ в направлении оси $z$, на собственное подпространство одной из компонент $P_{\alpha}(\alpha=x$ или $\alpha=y$ ) оператора псевдомомента системы (а при $Q=0$ - на собственное подпространство обеих компонент: при $Q \neq 0$ берется лишь одна компонента, ибо $P_{x} P_{y} \neq P_{y} P_{x}$ ). Для гамильтониана системы $Z_{1}$ такой подход позволил при $Q=0$ и фиксированных $P_{x}$,

* Научно-исследовательский радиофизический институт, Н. Новгород, Россия. E-mail: greg@nirfi.sci-nnov.ru 
$P_{y}$ изучить структуру сушественного и дискретного спектра, а при $Q \neq 0$ найти сушественный спектр. Однако для $Q \neq 0$ не были решены следующие важные задачи (а часть из них - и для $Q=0$ ).

I. О возможной зависимости спектра гамильтониана системы $Z_{1}$ от $P_{\alpha}$.

II. O структуре спектра гамильтониана системы $Z_{1}$ без фиксации $P_{\alpha}$ при $Q \neq 0$.

III. O структуре спектра гамильтонианов двухкластерных систем $Z_{2}=\left(C_{1}, C_{2}\right)$, получаюшихся из $Z_{1}$ после распадения из состояния с фиксированным $P_{\alpha}$.

IV. O структуре чисто точечного спектра оператора системы $Z_{1}$ при фиксации $P_{\alpha}$ или без нее.

Наиболее важной является задача IV, ибо ее решение отвечает на вопрос о спектре связанных состояний системы. Но структура этого спектра сушественно зависит от свойств нижней границы спектра гамильтонианов составных систем $Z_{2}=\left(C_{1}, C_{2}\right)$ для тех распадений $Z_{2}$ системы $Z_{1}$, которые определяют нижнюю границу существенного спектра $Z_{1}$. Поэтому решение задачи IV требует предварительного решения задачи III. При этом задачу III надо решать без фиксации псевдомоментов кластеров, ибо фиксация компоненты $P_{\alpha}$ псевдомомента системы оставляет свободными компоненты $P_{\alpha}\left[C_{k}\right]$ псевдомоментов кластеров $C_{k}, k=1,2$, фиксируя лишь их сумму. Но спектр квантовых систем в однородном магнитном поле без фиксации псевдомомента вообше не изучался ни для распавшейся системы, ни даже для исходной. Поэтому необходимой ступенью для выработки подходов к задаче III является решение задачи II, имеюшее к тому же немалый самостоятельный интерес как первый результат о спектре гамильтонианов с магнитным полем без фиксации псевдомомента. Наконец, очевидно, что для решения задачи II в обшем случае достаточно решить задачу I. Таким образом, для решения задачи IV о структуре спектра связанных состояний системы необходимо предварительно решить задачи I, II и III.

Решение задач I-III является целью настояшей работы. При этом мы модифицируем подход, предложенный авторами статьи [1]. Вместо фиксации одной из компонент псевдомомента $\left(P_{x}\right.$ или $\left.P_{y}\right)$, что неоправданно выделяло бы одну из координат, мы фиксируем их сумму $P_{\Sigma}=P_{x}+P_{y}$, т. е. сужаем исходньй оператор на собственное подпространство оператора $P_{\Sigma}$.

Главные результаты статьи:

1. Найден сушественный спектр системы $Z_{1}$ при фиксации $P_{\Sigma}$ и показано, что он не зависит от $P_{\Sigma}$.

2. Доказано, что спектр $Z_{1}$ без фиксации $P_{\Sigma}-$ тот же, что и при фиксации, но имеет дополнительное бесконечное вырождение ${ }^{1)}$.

3. Установлена структура спектра оператора энергии системы $Z_{2}=\left(C_{1}, C_{2}\right)$ при фиксации $P_{\Sigma}$ и без нее.

Для получения этих результатов мы применяем геометрические методы [2], [3], не повторяя здесь их стандартную часть, связанную с оценками энергии рассматриваемых систем в областях, отвечающих различным распадениям системы $Z_{1}$. В то же время мы подробно описываем новые идеи, относяшиеся к перестройке кинетической части операторов энергии, и их реализацию в соответствующих оценках.

\footnotetext{
1) Это принципиально отличает задачи с однородным магнитным полем от задач без поля, где отсутствие фиксации хотя бы одной компоненты момента приводит к замазыванию спектра относительного движения, а не к его дополнительному бесконечному вырождению.
} 
Результаты данной статьи были частично анонсированы в работе [4].

В заключение заметим, что выше мы говорили о фиксации суммы компонент псевдомомента, однако фактически в работе мы делаем следующую - эквивалентную - процедуру: после подходящего калибровочного преобразования мы фиксируем сумму компонент момента. Такой подход не дает преимушеств при рассмотрении исходного гамильтониана, но облегчает исследование спектров операторов составных систем $Z_{2}$.

\section{2. ОПРЕДЕЛЕНИЯ И ОСНОВНЫЕ РЕЗУЛЬТАТЫ}

2.1. Пусть $Z_{1}=\{1,2, \ldots, n\}$ - квантовая система $n$ частиц с конечными массами $m_{j}$, зарядами $e_{j}$ и координатами $r_{j}=\left(x_{j}, y_{j}, z_{j}\right)$, находяшаяся в однородном магнитном поле $\mathcal{F}=(0 \div 0.2 B)$, где $B>0$ - произвольная константа. Оператор энергии системы $Z_{1}$ имеет вид

$$
\mathcal{H}=\sum_{j=1}^{n} \frac{1}{m_{j}}\left(\frac{1}{i} \nabla_{j}-e_{j} A_{j}\right)^{2}+\frac{1}{2} \sum_{s, t=1 ; s \neq t}^{n} V_{s t}\left(r_{s t}\right),
$$

где $\nabla_{j}=\left(\partial / \partial x_{j}, \partial / \partial y_{j}, \partial / \partial z_{j}\right), A_{j}=B\left(-y_{j}, x_{j}, 0\right)$ - магнитный вектор-потенциал, $r_{i j}=r_{i}-r_{j}, V_{s t}\left(r_{s t}\right)=V_{t s}\left(r_{t s}\right)$ - потенциалы взаимодействия частиц. Мы предполагаем, что они вешественны и удовлетворяют условиям $\left.{ }^{2}\right)$

$$
\text { a) } V_{s t}\left(r_{1}\right) \in \mathcal{L}_{2, \text { lос }}\left(R^{3}\right), \quad \text { б) } V_{s t}\left(r_{1}\right) \rightarrow 0 \text { при }\left|r_{1}\right| \rightarrow \infty .
$$

В данной работе рассматриваются только заряженные системы, т.е. предполагается, что

$$
Q=\sum_{j=1}^{n} e_{j} \neq 0
$$

2.2. Пусть $M=\sum_{j=1}^{n} m_{j}, r_{\mathrm{c}}=\left(\alpha_{01}, \alpha_{02}, \alpha_{03}\right)$, где $\alpha_{01}=\sum_{j=1}^{n} m_{j} x_{j} M^{-1}, \alpha_{02}=$ $\sum_{j=1}^{n} m_{j} y_{j} M^{-1}, \quad \alpha_{03}=\sum_{j=1}^{n} m_{j} z_{j} M^{-1}$. Очевидно, что $r_{c}-$ радиус-вектор центра масс системы $Z_{1}$. Введем в (2.1) относительные координаты $q_{j}=\left(q_{j 1}, q_{j 2}, q_{j 3}\right):=r_{j}-r_{\mathrm{c}}$, координаты $\xi_{0}=\left(\alpha_{01}-\alpha_{02}\right) / 2, \eta_{0}=\left(\alpha_{01}+\alpha_{02}\right) / 2, \zeta_{0}=\alpha_{03}$ и отделим движение центра масс $Z_{1}$ в направлении оси $z$. Тогда мы получим оператор энергии относительного движения системы $Z_{1}$ в направлении оси $z$

$$
\mathcal{H}_{0}=T_{\perp}+T_{03}+V(q),
$$

где

$$
\begin{gathered}
T_{\perp}=\sum_{j=1}^{n} \sum_{p=1}^{2} \frac{1}{m_{j}}\left[B_{j p}+\frac{m_{j}}{2 M i}\left(\frac{\partial}{\partial \eta_{0}}-(-1)^{p} \frac{\partial}{\partial \xi_{0}}\right)+\right. \\
\left.+(-1)^{p+1} B e_{j} q_{j \bar{p}}+B e_{j}\left((-1)^{p+1} \eta_{0}-\xi_{0}\right)\right]^{2}, \\
T_{03}=\sum_{j=1}^{n} \frac{1}{m_{j}} B_{j 3}^{2}, \quad B_{j p}=\frac{1}{i} \sum_{s=1}^{n} \frac{\partial}{\partial q_{s p}}\left(\delta_{s j}-\frac{m_{j}}{M}\right), \\
V(q)=\frac{1}{2} \sum_{s, t=1 ; s \neq t}^{n} V_{s t}\left(q_{s}-q_{t}\right), \quad q=q_{1}, \ldots, q_{n},
\end{gathered}
$$

\footnotetext{
2) Условие (2.1б) может быть несколько ослаблено, но мы не стремимся здесь к максимальной общности.
} 
$\bar{p}=1$ при $p=2, \bar{p}=2$ при $p=1$.

Оператор $\mathcal{H}_{0}$ определен в $(3 n-1)$-мерном пространстве $\bar{R}_{0}^{(3 n-1)}$ переменных $q, \xi_{0}, \eta_{0}$, где $\sum_{j=1}^{n} m_{j} q_{j}=0$.

Далее с помощью подходящего калибровочного преобразования приведем оператор $\mathcal{H}_{0}$ к такому виду, в котором коэффициент перед $\eta_{0}$ равен нулю. Положим $D_{ \pm}=D_{ \pm}(q)=$ $B \sum_{j=1}^{n} e_{j}\left(q_{j 1} \pm q_{j 2}\right), \omega_{0}=D_{-}+2 Q B \xi_{0}$,

$$
\mathcal{H}\left(\frac{\partial}{i \partial \eta_{0}}\right)=e^{i \eta_{0} \omega_{0}} \mathcal{H}_{0} e^{-i \eta_{0} \omega_{0}}
$$

Очевидно, что

$$
\mathcal{H}\left(\frac{\partial}{i \partial \eta_{0}}\right)=T_{\perp}\left(\frac{\partial}{\partial \eta_{0}}\right)+T_{03}+V(q)
$$

где

$$
\begin{gathered}
T_{\perp}\left(\frac{\partial}{\partial \eta_{0}}\right)=\sum_{j=1}^{n} \sum_{p=1}^{2} \frac{1}{m_{j}}\left(A_{i p}+h_{j p 0}+\frac{m_{j}}{2 M i} \frac{\partial}{\partial \eta_{0}}\right)^{2}, \\
h_{j p 0}=\frac{m_{j}}{2 M i}(-1)^{p+1} \frac{\partial}{\partial \xi_{0}}-F_{j} \xi_{0}, \\
A_{j p}=B_{j p}-\frac{m_{j} D_{-}}{2 M}+(-1)^{p+1} B e_{j} q_{j \bar{p}}, \quad F_{j}=B e_{j}+\frac{B Q m_{j}}{M} .
\end{gathered}
$$

Поэтому

$$
\int \mathcal{H}_{0}\left(\frac{\partial}{i \partial \eta_{0}}\right) e^{-i \eta_{0} \lambda_{0}} d \eta_{0}=\mathcal{H}_{0}\left(\lambda_{0}\right), \quad \lambda \in R^{1},
$$

где оператор $\mathcal{H}_{0}\left(\lambda_{0}\right)$ определяется равенством $(2.4)$, если $\partial /\left(i \partial \eta_{0}\right)$ заменить на $\lambda_{0}$. По построению оператор $\mathcal{H}_{0}\left(\lambda_{0}\right)$ в пространстве $R_{0}^{(3 n-1)}=\left\{q, \xi_{0}, \lambda_{0}\right\}$ унитарно эквивалентен оператору $\mathcal{H}_{0}$ в $\bar{R}_{0}^{(3 n-1)}$

2.3. Отметим, что перейти от оператора (2.3) к оператору (2.5) можно было иначе, используя подход, связанный с оператором псевдомомента [1]. Действительно, пусть $P=\left(P_{x}, P_{y}\right)$ - оператор псевдомомента системы $Z_{1}$ (выражения для $P_{x}, P_{y}$ см. в работе [5]). Обозначим через $U_{2 \lambda_{0}}$ собственное подпространство оператора $P_{\Sigma}=\left(P_{x}+\right.$ $\left.P_{y}\right) / 2$, отвечаюшее его собственному значению $2 \lambda_{0}$. Легко видеть, что $U_{2 \lambda_{0}}=\{\tilde{\psi}\}$, где $\tilde{\psi}=e^{-i \eta_{0}\left(\omega_{0}+\lambda_{0}\right)} \psi\left(q, \xi_{0}, \lambda_{0}\right)$ и $\psi-$ произвольная функция из области определения $P_{\Sigma}$. При $\tilde{\psi} \in U_{2 \lambda_{0}} \mathcal{H}_{0} \tilde{\psi}=e^{-i \eta_{0}\left(\omega_{0}+\lambda_{0}\right)} \mathcal{H}_{0}\left(\lambda_{0}\right) \psi\left(q, \xi_{0}, \lambda_{0}\right)$, где оператор $\mathcal{H}_{0}\left(\lambda_{0}\right)$ определяется формулой (2.5). Таким образом, исследование спектра оператора $\mathcal{H}_{0}$ после сужения его на собственное подпространство $U_{2 \lambda_{0}}$ оператора $P_{\Sigma}$ приводит к изучению спектра полученного ранее оператора $\mathcal{H}_{0}\left(\lambda_{0}\right)$ на функциях $\psi\left(q, \xi_{0}, \lambda_{0}\right)$ при фиксированном $\lambda_{0}$. Если $\lambda_{0}$ не фиксировать, то мы получим оператор, унитарно эквивалентный исходному.

2.4. Оператор $\mathcal{H}_{0}\left(\lambda_{0}\right)$ мы будем рассматривать как в пространстве $R_{0}^{(3 n-1)}=$ $\left\{q, \xi_{0}, \lambda_{0}\right\}$, так и в пространстве $R_{0}^{(3 n-2)}=\left\{q, \xi_{0}\right\}$ при фиксированном $\lambda_{0}$. В последнем случае вместо $\mathcal{H}_{0}\left(\lambda_{0}\right)$ мы будем писать $\widehat{\mathcal{H}}_{0}\left(\lambda_{0}\right)$. Операторы $\mathcal{H}_{0}, \mathcal{H}_{0}\left(\lambda_{0}\right)$ и $\widehat{\mathcal{H}}_{0}\left(\lambda_{0}\right)$ определим на функциях из $C_{0}^{2}$ соответственно в пространствах $\mathcal{L}_{2}\left(R_{0}^{-(3 n-1)}\right), \mathcal{L}_{2}\left(R_{0}^{(3 n-1)}\right)$ 
и $\mathcal{L}_{2}\left(R_{0}^{(3 n-2)}\right)$. Они будут полуограничены снизу, и мы расширим их до самосопряженных, сохраняя прежние обозначения. Всюду далее обозначим через $\sigma_{\mathrm{ess}}(W), \sigma_{\mathrm{d}}(W)$, $\sigma_{\mathrm{pp}}(W), \sigma_{\mathrm{p}}(W)$ и $\sigma(W)$ соответственно сушественный, дискретный, чисто точечный, точечный и весь спектр произвольного оператора $W ; D_{W}$ - область определения $W$.

TEOPEмa 2.1. Cпектр $\sigma\left(\widehat{\mathcal{H}}_{0}\left(\lambda_{0}\right)\right)$ не зависит от $\lambda_{0}$. Cnектр $\sigma\left(\mathcal{H}_{0}\right)$ совпадает с $\sigma\left(\widehat{\mathcal{H}}\left(\lambda_{0}\right)\right)$, однако кажсдая точка $\nu$ из $\sigma\left(\mathcal{H}_{0}\right)$ имеет дополнительное бесконечное вырождение по сравнению с этой жсе точкой из $\sigma\left(\hat{\mathcal{H}}_{0}\left(\lambda_{0}\right)\right)$.

СлЕДСТВИЕ 2.1. Дискретный спектр $\sigma_{\mathrm{d}}\left(\mathcal{H}_{0}\right)$ всегда пуст.

СЛЕДСТВИЕ 2.2. Каждая точка из $\sigma_{\mathrm{p}}\left(\widehat{\mathcal{H}}_{0}\left(\lambda_{0}\right)\right)$ является бесконечно вырожденным собственным значением оператора $\mathcal{H}_{0}$, причем если $\nu \in \sigma_{\mathrm{d}}\left(\widehat{\mathcal{H}}_{0}\left(\lambda_{0}\right)\right)$, то $\nu \in \sigma_{\mathrm{pp}}\left(\mathcal{H}_{0}\right)$.

В силу теоремы 2.1 достаточно изучать далее спектр оператора

$$
H=\widehat{\mathcal{H}}_{0}(0)=T_{\perp}(0)+T_{03}+V(q) .
$$

2.5. Для описания спектра $H$ нам понадобятся операторы $H\left(Z_{k}\right)$ составных систем $Z_{k}=\left(C_{1}, \ldots, C_{k}\right)$, получающихся из $Z_{1}$ разбиением на $k, 2 \leqslant k \leqslant n$, не взаимодействующих между собой кластеров $C_{i}\left(Z_{1}=\bigcup_{j=1}^{k} C_{j}, C_{j} \cap C_{i}=\varnothing, j \neq i\right)$. Пусть

$$
\begin{gathered}
H\left(Z_{k}\right)=H-\frac{1}{2} \sum_{i, j=1, i \neq j}^{k} \sum_{s \in C_{i}, t \in C_{j}} V_{s t}\left(q_{s}-q_{t}\right), \\
\mu=\min _{Z_{k}, k \geqslant 2} \inf H\left(Z_{k}\right) .
\end{gathered}
$$

Если $\widetilde{Z}_{k-1}=\left(C_{1}, \ldots, C_{k-2}, \widetilde{C}_{k-1}\right)$, где $\widetilde{C}_{k-1}=C_{k-1} \cup C_{k}$, то очевидно, что $\inf H\left(\widetilde{Z}_{k-1}\right) \leqslant \inf H\left(Z_{k}\right)$. Поэтому в $(2.8)$ можно брать лишь $k=2$, т.е.

$$
\mu=\min _{Z_{2}} \inf H\left(Z_{2}\right)
$$

TеОРема 2.2. Существенный спектр $\sigma_{\mathrm{ess}}(H)$ состоит из всех точек луча $[\mu,+\infty)$.

Теорема 2.2 описывает структуру существенного спектра $\sigma_{\mathrm{ess}}(H)$ в обычной ХВЖКформулировке. Эквивалентный результат для оператора, вычисленного при фиксации одной компоненты псевдомомента, получен в работе [1] другим методом и в значительно более сложной формулировке.

2.6. Пусть $O^{\prime}=\left\{Z_{2} \mid Z_{2}=\left(C_{1}, C_{2}\right)\right.$, inf $\left.H\left(Z_{2}\right)=\mu\right\}$.

При изучении $\sigma_{\mathrm{d}}(H)$ важно знать свойства нижней границы спектра операторов $H_{0}\left(Z_{2}\right), Z_{2} \in O^{\prime}$, полученных из операторов $H\left(Z_{2}\right)$ после введения относительных координат частищ в кластерах $C_{j}$ и отделения движения ЦМ этих кластеров в направлении оси $z$. Далее мы выпишем явные выражения операторов $H_{0}\left(Z_{2}\right)$ и сфформулируем результаты работы о спектре $\sigma\left(H_{0}\left(Z_{2}\right)\right)$. 
Пусть $Z_{2}=\left(C_{1}, C_{2}\right), \quad M_{j}=\sum_{s \in C_{j}} m_{s}, \quad Q_{j}=\sum_{s \in C_{j}} e_{s}, \quad \alpha_{j}=\left(\alpha_{j 1}, \alpha_{j 2}, \alpha_{j 3}\right)=$ $\sum_{s \in C_{j}} m_{s} q_{s} M_{j}^{-1}$, т.е. $M_{j}, Q_{j}, \alpha_{j}$ - масса, заряд и радиус-вектор ЦМ кластера $C_{j}$, соответственно. Введем относительные координаты $q^{(1)}=\left(q_{1}^{(1)}, \ldots, q_{n}^{(1)}\right)$ частиц в кластерах $C_{j}$

$$
q_{s}^{(1)}=\left(q_{s 1}^{(1)}, q_{s 2}^{(1)}, q_{s 3}^{(1)}\right)=q_{s}-\alpha_{j}, \quad s \in C_{j},
$$

и, кроме того, положим

$$
\xi_{1}=\frac{\alpha_{11}-\alpha_{12}}{2}, \quad \eta_{1}=\frac{\alpha_{11}+\alpha_{12}}{2}, \quad \zeta_{1}=\alpha_{13}-\alpha_{23} .
$$

Ясно, что

$$
\sum_{s \in C_{j}} m_{s} q_{s}^{(1)}=0, \quad j=1,2 .
$$

Кроме того, поскольку $\sum_{j=1}^{n} m_{j} q_{j}=0$, то $M_{1} \alpha_{1}+M_{2} \alpha_{2}=0$ и $\alpha_{2}=-M_{1} M_{2}^{-1} \alpha_{1}$. С учетом этого

$$
\begin{aligned}
& q_{s 1}=q_{s 1}^{(1)}+\xi_{1}+\eta_{1}, \quad q_{s 2}=q_{s 2}^{(1)}+\eta_{1}-\xi_{1}, \\
& q_{s 3}=q_{s 3}^{(1)}+M_{2} M^{-1} \zeta_{1}, \quad \text { если } s \in C_{1}, \\
& q_{s 1}=q_{s 1}^{(1)}-M_{1} M_{2}^{-1}\left(\eta_{1}+\xi_{1}\right), \quad q_{s 2}=q_{s 2}^{(1)}-M_{1} M_{2}^{-1}\left(\eta_{1}-\xi_{1}\right), \\
& q_{s 3}=q_{s 3}^{(1)}-M_{1} M \zeta_{1}, \quad \text { если } s \in C_{2} .
\end{aligned}
$$

В новых координатах

$$
H\left(Z_{2}\right)=H_{0}\left(Z_{2}\right)+H_{c}\left(Z_{2}\right)
$$

где

$$
\begin{gathered}
H_{0}\left(Z_{2}\right)=T\left(Z_{2}\right)+V_{Z_{2}}, \quad H_{c}\left(Z_{2}\right)=-M M_{1}^{-1} M_{2}^{-1} \frac{d^{2}}{d \zeta_{1}^{2}} \\
T\left(Z_{2}\right)=\sum_{k=1}^{2} T\left[C_{k}\right], \quad V_{Z_{2}}=\sum_{k=1}^{2} V\left[C_{k}\right], \quad T\left[C_{k}\right]=T_{\perp}\left[C_{k}\right]+T_{03}\left[C_{k}\right] \\
T_{\perp}\left[C_{k}\right]=\sum_{j \in C_{k}} \sum_{p=1}^{2} m_{j}^{-1}\left\{\tilde{A}_{j p}(k)+h_{j p 0}-(-1)^{k} \frac{m_{j} M_{2}}{2 M M_{k} i}\left[(-1)^{p+1} \frac{\partial}{\partial \xi_{1}}+\frac{\partial}{\partial \eta_{1}}\right]+\right. \\
+(-1)^{k} B e_{j} M_{1} M_{k}^{-1}\left[\xi_{1}+(-1)^{p} \eta_{1}\right]- \\
\left.-m_{j} M^{-1} M_{2}^{-1}\left(Q_{1} M_{2}-Q_{2} M_{1}\right) B \xi_{1}\right\}^{2}, \\
T_{03}\left[C_{k}\right]=\sum_{j \in C_{k}} m_{j}^{-1} B_{j 3}^{2}(k), \\
\tilde{A}_{j p}(k)=B_{j p}(k)-\frac{1}{2} m_{j} M^{-1} D_{-}\left(q^{(1)}\right)-(-1)^{p} B e_{j} q_{j \bar{p}}^{(1)}, \quad p=1,2, \\
B_{j p}(k)=\frac{1}{i} \sum_{s \in C_{k}} \frac{\partial}{\partial q_{s p}^{(1)}}\left(\delta_{s j}-\frac{m_{j}}{M_{k}}\right), \quad p=1,2,3 \\
V\left[C_{k}\right]=\frac{1}{2} \sum_{s, t \in C_{k}, s \neq t} V_{s t}\left(q_{s}^{(1)}-q_{t}^{(1)}\right) .
\end{gathered}
$$


Если один из кластеров, скажем $C_{2}$, состоит из единственной частицы, например, с номером $t$, то выражение оператора $H_{0}\left(Z_{2}\right)$ и все полученные из него упрошаются, ибо тогда $\alpha_{2}=q_{t}, q_{t}^{(1)}=(0,0,0) ;$ следовательно, производные по $q_{t p}^{(1)}$ будут отсутствовать и $\tilde{A}_{t p}(2)=V\left[C_{2}\right]=0 . \quad$ Оператор $H_{0}\left(Z_{2}\right)$ определен в пространстве $\bar{R}_{0}^{(3 n-3)}=$ $\left\{q, \xi_{0}, \xi_{1}, \eta_{1}\right\}$. Преобразуем его аналогично оператору $\mathcal{H}_{0}$. Найдем сначала такую функцию $\omega_{1}$, что

$$
H_{0}\left(Z_{2}, \frac{\partial}{i \partial \eta_{1}}\right):=e^{i \eta_{1} \omega_{1}} H_{0}\left(Z_{2}\right) e^{-i \eta_{1} \omega_{1}}
$$

не будет содержать членов вида $c \eta_{1}$, а потом рассмотрим оператор

$$
\int H_{0}\left(Z_{2}, \frac{\partial}{i \partial \eta_{1}}\right) e^{-i \lambda_{1} \eta_{1}} d \eta_{1}=H_{0}\left(Z_{2}, \lambda_{1}\right) .
$$

Пусть

$$
\begin{gathered}
D_{ \pm}\left(q^{(1)}, k\right)=(-1)^{k+1} M_{1} M_{k}^{-1} B \sum_{t \in C_{k}} e_{t}\left(q_{t_{1}}^{(1)} \pm q_{t 2}^{(1)}\right), \\
\gamma_{k-1}=2 B Q_{1}+2(-1)^{k} B Q_{2} M_{1}^{2} M_{k}^{-1} M^{-1}, \quad k=1,2 .
\end{gathered}
$$

Тогда при $\omega_{1}=D_{-}\left(q^{(1)}, 1\right)+D_{-}\left(q^{(1)}, 2\right)+\gamma_{0} \xi_{0}+\gamma_{1} \xi_{1}$ оператор $(2.14)$ не будет содержать членов с $\eta_{1}$ и

$$
H_{0}\left(Z_{2}, \lambda_{1}\right)=\sum_{k=1}^{2}\left(T\left[C_{k}, \lambda_{1}\right]+V\left[C_{k}\right]\right)
$$

где

$$
\begin{gathered}
T\left[C_{k}, \lambda_{1}\right]=T_{\perp}\left[C_{k}, \lambda_{1}\right]+T_{03}\left[C_{k}\right], \\
T_{\perp}\left[C_{k}, \lambda_{1}\right]=\sum_{j \in C_{k}} \sum_{p=1}^{2} \frac{1}{m_{j}}\left\{A_{j p}(k)+\sum_{s=0}^{1} h_{j p s}(k)+(-1)^{k+1} \frac{m_{j} M_{2}}{2 M_{k} M} \lambda_{1}\right\}^{2}, \\
h_{j p 0}(k)=(-1)^{p+1} \frac{1}{i} \frac{m_{j}}{2 M} \frac{d}{d \xi_{0}}-F_{j}(k) \xi_{0}, \\
h_{j p 1}(k)=(-1)^{k} \frac{M_{1}}{M_{k}}\left(\frac{1}{2 i} \frac{m_{j} M_{2}}{M_{1} M}(-1)^{p} \frac{d}{d \xi_{1}}+F_{j}(k) \xi_{1}\right), \\
F_{j}(k)=e_{j}+\frac{Q_{k}}{M_{k}} m_{j}, \quad A_{j p}(k)=B_{j p}(k)-m_{j}\left(2 M_{k}\right)^{-1} D_{-}\left(q^{(1)}, k\right)-(-1)^{p} B e_{j} q_{j \bar{p}}^{(1)} .
\end{gathered}
$$

Оператор $H_{0}\left(Z_{2}, \lambda_{1}\right)$ определен в пространстве $R_{0}^{(3 n-3)}=\left\{q^{(1)}, \xi_{0}, \xi_{1}, \lambda_{1}\right\}$ и по построению унитарно эквивалентен $H_{0}\left(Z_{2}\right)$.

Если оператор $H_{0}\left(Z_{2}, \lambda_{1}\right)$ будет рассматриваться в пространстве $R_{0}^{(3 n-4)}=$ $\left\{q^{(1)}, \xi_{0}, \xi_{1}\right\}$ при фиксированном $\lambda_{1}$, то мы будем обозначать его через $\widehat{H}_{0}\left(Z_{2}, \lambda_{1}\right)$.

2.7. Следующие теоремы (2.3 и 2.4) описывают спектральные свойства операторов $H_{0}\left(Z_{2}\right)$ и $\widehat{H}_{0}\left(Z_{2}, \lambda_{1}\right)$ распадений $Z_{2}=\left(C_{1}, C_{2}\right)$, для которых

$$
Q_{1} Q_{2} \neq 0
$$


Tеорема 2.3. Cпектр $\sigma\left(\widehat{H}_{0}\left(Z_{2}, \lambda_{1}\right)\right)$ не зависит от $\lambda_{1}$. Cпектр $\sigma\left(H_{0}\left(Z_{2}\right)\right)$ совпадает со спектром $\sigma\left(\widehat{H}_{0}\left(Z_{2}, 0\right)\right)$, однако каждая точка $\nu \in \sigma\left(H_{0}\left(Z_{2}\right)\right)$ имеет дополнительное бесконечное вырождение по сравнению $с$ этой жсе точкой из $\sigma\left(\widehat{H}_{0}\left(Z_{2}, 0\right)\right)$.

СлЕДСТВИЕ 2.3. Спектр $\sigma_{\mathrm{d}}\left(H_{0}\left(Z_{2}\right)\right)$ всегда nycm.

СлЕДСТВИЕ 2.4. Каждая точка $\nu$ из $\sigma_{\mathrm{p}}\left(\widehat{H}_{0}\left(Z_{2}, 0\right)\right)$ является бесконечно вирожденным собственным значением оператора $H_{0}\left(Z_{2}\right)$, причем если $\nu \in$ $\sigma_{\mathrm{d}}\left(\widehat{H}_{0}\left(Z_{2}, 0\right)\right)$, mo $\nu \in \sigma_{\mathrm{pp}}\left(H_{0}\left(Z_{2}\right)\right)$.

СлЕДСТВиЕ 2.5. $\inf H_{0}\left(Z_{2}\right)=\inf \widehat{H}_{0}\left(Z_{2}, 0\right)$.

2.8. Для произвольного фиксированного $Z_{2}=\left(C_{1}, C_{2}\right)$ обозначим через $Z_{3}^{\prime}=$ $\left(C_{1}^{\prime}, C_{2}^{\prime}, C_{3}^{\prime}\right)$ любое разбиение исходной системы $Z_{3}$, получаюшееся из $Z_{2}^{\prime}$ дроблением какого-либо из кластеров $C_{k}$, и положим

$$
G\left(Z_{3}^{\prime}\right)=\widehat{H}_{0}\left(Z_{2}, 0\right)-\sum_{s \in C_{i}^{\prime} ;, t \in C_{j}^{\prime}} V_{s t}\left(q_{s}^{(1)}-q_{t}^{(1)}\right), \quad \mu\left(Z_{2}\right)=\min _{Z_{3}^{\prime}} \inf G\left(Z_{3}^{\prime}\right),
$$

где $C_{i}^{\prime} \cup C_{j}^{\prime}=C_{k}, C_{k}$ - тот кластер из $\in Z_{2}$, дроблением которого получалось $Z_{3}^{\prime}$.

TЕОРема 2.4. Существенныц спектр $\sigma_{\text {ess }}\left(\widehat{H}_{0}\left(Z_{2},\right)\right)$ совпадает $\left[\mu\left(Z_{2}\right),+\infty\right)$.

Теоремы $2.3,2.4$ служат фундаментом для исследования структуры спектра $\sigma_{\mathrm{d}}\left(H_{0}\right)$ (и совпадающего с ним бесконечно вырожденного спектра $\sigma_{\mathrm{pp}}(\mathcal{H})$ ). Дело в том, что доказательство того, что дискретный спектр гамильтонианов квантовых систем не пуст, сушественно облегчается, если $\mu \in \sigma_{\mathrm{p}}\left(H_{0}\left(Z_{2}\right)\right)$. А из теорем $2.3,2.4$ следует достаточное условие этого включения - неравенство

$$
\mu<\mu\left(Z_{2}\right)
$$

Разумеется, оно верно не всегда, но даже когда выполняется, доказать его весьма трудно. Мы займемся проверкой неравенства (2.18) для конкретных систем в отдельной работе.

\section{3. ДОКАЗАТЕЛЬСТВА ТЕОРЕМ 2.1-2.4}

3.1. Докажем теорему 2.1. Пусть $a_{s p}, \quad b-$ некоторые числа, $\varkappa=$ $\sum_{s=1}^{n} \sum_{p=1}^{2} a_{s p} q_{s p}$. Проведем калибровочное преобразование

$$
e^{i \varkappa \lambda_{0}} \mathcal{H}_{0}\left(\lambda_{0}\right) e^{-i \varkappa \lambda_{0}}
$$

и затем перейдем в операторе (3.1) к новым переменным, полагая

$$
q^{\prime}=q, \quad \lambda_{0}^{\prime}=\lambda_{0}, \quad \xi_{0}^{\prime}=\xi_{0}-b_{0} \lambda_{0} .
$$

Обозначим полученный оператор через $\mathcal{H}_{0}^{\prime}\left(\lambda_{0}^{\prime}\right)$. Элементарные выкладки показьвают, что

$$
\mathcal{H}_{0}^{\prime}\left(\lambda_{0}^{\prime}\right)=\sum_{j=1}^{n} \sum_{p=1}^{2}\left(A_{j p}^{\prime}+d_{j p} \lambda_{0}^{\prime}+h_{j p 0}^{\prime}\right)^{2}+T_{03}^{\prime}+V^{\prime}
$$


где штрихи указывают на замену $q \rightarrow q^{\prime}, \xi_{0} \rightarrow \xi_{0}^{\prime}$;

$$
d_{j p}=-\sum_{s=1}^{n} a_{s p}\left(\delta_{s j}-m_{j} M^{-1}\right)+m_{j}(2 M)^{-1}-b_{0} M^{-1}\left(Q m_{j}+M e_{j}\right) B .
$$

Покажем, что выбором чисел $a_{s p}$ и $b_{0}$ можно добиться выполнения равенств

$$
d_{j p}=0
$$

при $j=1,2, \ldots, n, p=1,2$.

Суммируя (3.4) по $j$, видим, что $1 / 2-2 b_{0} B Q=0$, т.е. $b_{0}=(4 B Q)^{-1}$. Подставив это выражение в (3.4), получим систему уравнений

$$
a_{j p}-m_{j} M^{-1} \sum_{s=1}^{n} a_{s p}+m_{j}(4 M)^{-1}-e_{j}(4 Q)^{-1}=0, \quad j=1,2, \ldots, n, \quad p=1,2 .
$$

Будем искать $a_{j p}$ в виде $a_{j p}=a e_{j}+\tilde{a} m_{j}$, где $a$ и $\tilde{a}-$ неизвестные числа. Тогда из последней системы уравнений получается, что $a=(4 Q)^{-1}, \tilde{a}$-произвольно; мы возьмем $\tilde{a}=0$. Таким образом, в результате калибровочного преобразования $(3.1)$ с $\varkappa=(4 B Q)^{-1} D_{+}$ и замены $(3.2)$ с $b_{0}=(4 B Q)^{-1}$ мы получим оператор (3.3) с $d_{j p}=0, j=1, \ldots, n, p=$ 1, 2. Этот оператор, если отвлечься от штрихов у переменных, совпадает с $\mathcal{H}(0)$. Поэтому далее мы будем рассматривать $\mathcal{H}(0)$. По построению $\mathcal{H}_{0}, \mathcal{H}\left(\lambda_{0}\right)$ и $\mathcal{H}(0)$ унитарно эквивалентны. Поскольку оператор $\mathcal{H}(0)$ не зависит от $\lambda_{0}$, то его спектры в $\mathcal{L}_{2}\left(R_{0}^{(3 n-1)}\right)$ и в $\mathcal{L}_{2}\left(R_{0}^{(3 n-2)}\right)$ совпадают, но спектр в $\mathcal{L}_{2}\left(R_{0}^{(3 n-1)}\right)$ имеет дополнительное бесконечное вырождение по сравнению со спектром в $\mathcal{L}_{2}\left(R_{0}^{(3 n-2)}\right)$. Наконец, если рассматривать оператор $\widehat{\mathcal{H}}_{0}\left(\lambda_{0}\right)$ (см. п. 2.4$)$, то аналогично предыдушему убеждаемся, что $\widehat{\mathcal{H}}_{0}\left(\lambda_{0}\right)$ унитарно эквивалентен $\mathcal{H}(0)$ в $\mathcal{L}_{2}\left(R_{0}^{(3 n-2)}\right)$. Теорема 2.1 доказана. Отметим, что для нейтральных систем (т.е. при $Q=0$ ) это доказательство не подходит, и утверждения теоремы 2.1, по-видимому, не верны.

3.2. Доказательство теоремы 2.2 состоит из двух частей. Пусть $H=\widehat{\mathcal{H}}_{0}(0)$. Труднее установить, что если $\nu \in \sigma_{\mathrm{ess}}(H)$, то $\nu \geqslant \mu$; более легко показать, что если $\nu \geqslant \mu$, то $\nu \in$ $\sigma_{\text {ess }}(H)$. Мы приведем доказательство того раздела “трудной” части, который является новым по сравнению с работой [2] (п. 3.3-3.8). Для “легкой” части ограничимся лиш описанием схемы доказательства.

Пусть $Z_{2}=\left(C_{1}, C_{2}\right) \in O^{\prime}$. Так как $\inf H_{0}\left(Z_{2}\right)=\inf H\left(Z_{2}\right)$, то $\mu \in \sigma\left(H_{0}\left(Z_{2}\right)\right)$. Поэтому для каждого $\varepsilon>0$ сушествует $\psi_{\varepsilon}\left(q, \xi_{0}, \eta_{0}\right),\left\|\psi_{\varepsilon}\right\|=1$ такая, что

$$
\left\|H_{0}\left(Z_{2}\right) \psi_{\varepsilon}-\mu \psi_{\varepsilon}\right\| \leqslant \varepsilon .
$$

Пусть $\nu>\mu, \delta^{2}=\nu-\mu, N>0$ и функция $\tilde{g}_{N}\left(\zeta_{1}\right)$ такова, что $\tilde{g}_{N} \in C_{0}^{2}, \quad \tilde{g}_{N}\left(\zeta_{1}\right)=$ $\sin \delta\left(M_{1} M_{2} M^{-1}\right)^{1 / 2} \zeta_{1}$ при $\left|\zeta_{1}\right| \leqslant N, \tilde{g}_{N}\left(\zeta_{1}\right) \equiv 0$ при $\left|\zeta_{1}\right|>N+1$ и

$$
\sup _{N}\left(\max _{\zeta_{1}}\left(\left|\tilde{g}_{N}\right|+\left|\tilde{g}_{N}^{\prime}\right|+\mid \tilde{g}_{N}^{\prime \prime}\right)\right)<+\infty \text {. }
$$

Положим $g_{N}=\tilde{g}_{N} /\left\|\tilde{g}_{N}\right\|, E_{N}=\{\zeta|N \leqslant| \zeta \mid \leqslant N+1\}$. Очевидно, что

$$
\left\|H_{c}\left(Z_{2}\right) g_{N}-\delta^{2} g_{N}\right\|=\left\|H_{c}\left(Z_{2}\right) g_{N}-\delta^{2} g_{N}\right\|_{E_{N}} \rightarrow 0
$$


при $N \rightarrow \infty$ ибо в силу (3.6) $\sup _{N}\left(\left\|H_{c}\left(Z_{2}\right) \tilde{g}_{N}\right\|_{E_{N}}+\left\|\tilde{g}_{N}\right\|_{E_{N}}\right)<+\infty$, a $\left\|\tilde{g}_{N}\right\| \rightarrow \infty$ при $N \rightarrow \infty$. Громоздкие оценки, которые мы здесь приводить не будем, показывают, что

$$
\lim _{N \rightarrow \infty}\left\|V_{s t} \psi_{\varepsilon} g_{N}\right\|=0, \quad s \in C_{i}, \quad t \in C_{j}, \quad i \neq j
$$

В силу (3.5), (3.7), (3.8), (2.7) и (2.12) $\varlimsup_{N \rightarrow \infty}\left\|H \psi_{\varepsilon} g_{N}-\nu \psi_{\varepsilon} g_{N}\right\| \leqslant \varepsilon$; так как значение $\varepsilon$ произвольно, то $\nu \in \sigma(H)$.

3.3. Пусть теперь $\nu \in \sigma_{\mathrm{ess}}(H)$. Докажем, что $\nu \geqslant \mu$. Так как $\nu \in \sigma_{\mathrm{ess}}(H)$, то сушествуют $g_{k}\left(q, \xi_{0}\right) \in D_{H}$ такие, что

$$
\lim _{k \rightarrow \infty}\left\|H g_{k}-\nu g_{k}\right\|=0, \quad g_{k} \rightarrow 0 \quad \text { в } \quad \mathcal{L}_{2}\left(R_{0}^{(3 n-2)}\right), \quad\left\|g_{k}\right\|=1 .
$$

Ясно, что

$$
\lim _{k \rightarrow \infty}\left(H g_{k}, g_{k}\right)=\nu
$$

Из последнего уравнения, (3.9) и теорем вложения Соболева вытекает, что последовательность $g_{k}$ компактна в $\mathcal{L}_{2}(\Omega)$ для любой ограниченной области $\Omega \subset R_{0}^{(3 n-2)}$. Отсюда и из (3.9) следует, что

$$
\lim _{k \rightarrow \infty}\left\|g_{k}\right\|_{\Omega}=0
$$

Легко видеть, что такую последовательность $g_{k}$ можно выбрать из $C_{0}^{2}$. Поэтому для справедливости неравенства $\nu \geqslant \mu$ достаточно доказать, что для любой последовательности $g_{k} \in C_{0}^{2}$, удовлетворяюшей $(3.9),(3.10)$ выполняется неравенство

$$
\underline{\lim }\left(H g_{k}, g_{k}\right) \geqslant \mu \text {. }
$$

3.4. Опишем общую идею доказательства (3.11), используя физическую терминологию. Равенство (3.10) означает, что волновые функции $g_{k}$ описьвают последовательность состояний системы, для которой вероятность нахождения всей системы $Z_{1}$ в любой ограниченной области $\Omega$ пространства $R_{0}^{(3 n-2)}$ стремится к нулю. Следовательно, $g_{k}$ описывает либо одно из распадений $Z_{s}=\left(C_{1}, \ldots, C_{s}\right)$ системы $Z_{1}$ на $s \geqslant 2$ кластеров или суперпозицию таких распадений, либо движение всей системы как целого. Ясно, что в первом случае для доказательства достаточно, грубо говоря, разложить состояние $g_{k}$ на состояния $g_{k}, Z_{s}$, отвечающие всевозможным распадениям $Z_{s}$ исходной системы, провести оценку снизу значений энергии системы по состояниям $g_{k, Z_{s}}$ и затем собрать вместе полученные оценки. В то же время во втором случае нет никаких шансов доказать, что $\nu \geqslant \mu$, ибо последовательность $g_{k}$ не отвечает распадению системы и значение энергии может быть меньше, чем минимальное из значений энергии по всем распадениям, т.е. ничто не запрещает неравенство $\nu=\lim \left(H g_{k}, g_{k}\right)<\mu$, противоположное (3.11). Таким образом, для доказательства (3.11) второй случай необходимо исключить. Пусть $b \gg 1$ - произвольное число, $\chi_{b}$ - характеристическая функция области $|q| \geqslant b$ в $R_{0}^{(3 n-2)}, \bar{\chi}_{b}=1-\chi_{b}$. Поскольку $\sum_{j=1}^{n} m_{j} q_{j}=0$, то $\sum_{s=1}^{n}\left|\sum_{j=1}^{n} m_{j}\left(q_{j}-q_{s}\right)\right|^{2}=M^{2}|q|^{2} \geqslant M^{2} b^{2}$ при $q \in \operatorname{supp} g_{k} \chi_{b}$. Поэтому при $q \in \operatorname{supp} g_{k} \chi_{b}$ и $b \rightarrow \infty$ хотя бы одна из величин $\left|q_{j}-q_{s}\right|^{2}$ стремится к бесконечности, т.е. последовательность $g_{k} \chi_{b}$ при больших $b$ описывает первый 
случай и, следовательно, для нее оценка вида (3.11) в принципе может быть доказана. Но $g_{k}=g_{k} \chi_{b}+g_{k} \bar{\chi}_{b}$, и для исключения второго случая достаточно показать, что $\lim _{k \rightarrow \infty}\left\|g_{k} \bar{\chi}_{b}\right\|=0$ при любом $b$, т.е. что по последовательности $g_{k}$ система уходит из области $|q|<b$ в область $|q|>b$, отвечаюшую первому случаю. Равенство $\lim _{k \rightarrow \infty}\left\|g_{k} \bar{\chi}_{b}\right\|=0$ не есть прямое следствие (3.10), ибо область $\operatorname{supp} \bar{\chi}_{b}$ не ограничена в $R_{0}^{(3 n-2)}$ из-за переменной $\xi_{0}$. Именно необходимость доказательства оценки $\lim _{k \rightarrow \infty}\left\|g_{k} \bar{\chi}_{b}\right\|=0$ для последовательности $g_{k}$ отличает задачи с магнитным полем, в которых полное отделение движения ЦМ невозможно, от задач без поля, в которых гамильтонианы и волновые функции зависят только от относительных координат $q$, область $|q| \leqslant b$ ограничена и соотношение (3.10) эквивалентно соотношению $\left\|g_{k} \bar{\chi}_{b}\right\| \rightarrow 0$ при $k \rightarrow \infty$.

3.5. Доказательство (3.10), (3.11) начнем с перестройки кинетической части гамильтониана $H$, отвечаюшей движению в плоскости $x, y$. Предварительно проведем простые алгебраические рассуждения, которые будут нужны и для доказательства теоремы 2.3. Пусть $n_{0}$ - натуральное число, $1 \leqslant n_{0} \leqslant n ; f_{j}=\left(f_{j 1}, f_{j 2}\right), \tilde{f}_{j}=\left(\tilde{f}_{j 1}, \tilde{f}_{j 2}\right)$, $j=1,2, \ldots, n_{0},-$ произвольные двумерные векторы, возможно с симметричными операторными компонентами; $f=\left(f_{1}, \ldots, f_{n_{0}}\right), \quad \tilde{f}=\left(\tilde{f}_{1}, \ldots, \tilde{f}_{n_{0}}\right) ; \mathcal{M}=\sum_{j=1}^{n_{0}} m_{j}$. Положим $\left(f_{j}, \tilde{f}_{j}\right)_{2}=\sum_{p=1}^{2} f_{j p} \tilde{f}_{j p},(f, \tilde{f})_{0}=\sum_{j=1}^{n_{0}} m_{j}\left(f_{j}, \tilde{f}_{j}\right)_{2}, f_{\mathrm{cp}}=\sum_{j=1}^{n_{0}} m_{j} f_{j p} \mathcal{M}^{-1}$, $f_{j}^{(\mathrm{c})}=f^{(\mathrm{c})}=\left(f_{\mathrm{c} 1}, f_{\mathrm{c} 2}\right), f_{j}^{(0)}=f_{j}-f^{(\mathrm{c})}, f_{\mathrm{c}}=\left(f_{1}^{(\mathrm{c})}, \ldots, f_{n_{0}}^{(\mathrm{c})}\right), f_{0}=\left(f_{1}^{(0)}, \ldots, f_{n_{0}}^{(0)}\right)$. Очевидно, что $f=f_{0}+f_{\mathrm{c}}, \sum_{j=1}^{n_{0}} m_{j} f_{j}^{(0)}=0$ и $\left(f_{\mathrm{c}}, f_{0}\right)_{0}=0$. Поэтому

$$
(f, f)_{0}=\left(f_{0}, f_{0}\right)_{0}+\left(f_{\mathrm{c}}, f_{\mathrm{c}}\right)_{0}=\sum_{j=1}^{n_{0}} \sum_{p=1}^{2} m_{j}\left(f_{j p}-f_{\mathrm{c} p}\right)^{2}+\mathcal{M} \sum_{p=1}^{2} f_{\mathrm{c} p}^{2} .
$$

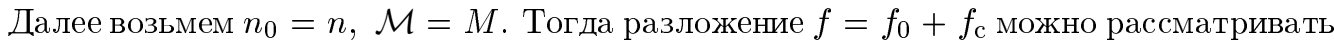
как разбиение $f$ на два "ортогональных" слагаемых, принадлежаших пространствам “относительного движения" ( $\left.f_{0}\right)$ и “движения центра масс" $\left(f_{\mathrm{c}}\right)$.

3.6. Применим (3.12) для перестройки оператора (2.6). Пусть $f_{j p}=\left(A_{j p}+h_{j p_{0}}\right) / m_{j}$. Тогда $T_{\perp}(0)=(f, f)_{0}$ и в силу $(3.12)$

$$
H=T_{0 \perp}+T_{\mathrm{c} \perp}+T_{03}+V,
$$

где

$$
\begin{gathered}
T_{0 \perp}=\sum_{j=1}^{n} \sum_{p=1}^{2}\left(f_{j p}-f_{\mathrm{c} p}\right)^{2} m_{j}, \quad T_{\mathrm{c} \perp}=M\left(f_{\mathrm{c} 1}^{2}+f_{\mathrm{c} 2}^{2}\right), \\
f_{\mathrm{c} p}=\left(h_{\mathrm{c} p_{0}}-\frac{1}{2} D_{-}-(-1)^{p} B \sum_{j=1}^{n} e_{j} q_{j \bar{p}}\right), \quad h_{\mathrm{c} p}=\frac{(-1)^{p+1}}{2 i} \frac{d}{d \xi_{0}}-2 B Q \xi_{0} .
\end{gathered}
$$

3.7. Используя (3.13), докажем сначала (3.10). В силу неравенств подчинения [5]

$$
\inf \left\{2^{-1}\left(T_{0 \perp}+T_{03}+T_{\mathrm{c} \perp}\right)+V\right\}>-\infty .
$$

Поэтому из условия $\lim _{k \rightarrow \infty}\left(H g_{k}, g_{k}\right)=\nu$ вытекает, что

$$
\varlimsup
$$

4 Теоретическая и математическая физика, т. 133, № 1, 2002 г. 
и, значит,

$$
\sup _{k}\left(\left\|\left(f_{j p}-f_{c p}\right) g_{k}\right\|^{2}+\left\|f_{c p} g_{k}\right\|^{2}+\left\|B_{j 3} g_{k}\right\|^{2}\right)<+\infty
$$

при любых $j, p$, поскольку операторы $f_{j p}, f_{\text {с } p}$ и $B_{j 3}$ симметричны. Поэтому для любой ограниченной области $\Omega \subset R_{0}^{(3 n-2)}$

$$
\sup _{k}\left\|\frac{\partial g_{k}}{\partial \xi_{0}}\right\|_{\Omega}<+\infty, \quad \sup _{k}\left\|B_{j p} g_{k}\right\|_{\Omega}<+\infty, \quad 1 \leqslant j \leqslant n, \quad p=1,2,3 .
$$

Операторы $B_{j p}$ записаны в зависимых координатах $q_{1}, \ldots, q_{n}$. Выберем в качестве независимых координат $q_{j}^{\prime}=q_{j}, j=1, \ldots, n-1$. Тогда

$$
q_{n p}=-\sum_{s=1}^{n-1}\left(m_{s} q_{s p}^{\prime}\right) m_{n}^{-1}, \quad B_{j p}=B_{j p}^{\prime}=\sum_{s=1}^{n-1} \frac{\partial}{\partial q_{s p}^{\prime}}\left(\delta_{s j}-\frac{m_{j}}{M}\right) .
$$

В силу (3.15)

$$
\sup _{k}\left\|B_{j p}^{\prime} g_{k}^{\prime}\right\|_{\Omega^{\prime}}<+\infty, \quad j=1, \ldots, n-1,
$$

где $g_{k}^{\prime}\left(q_{1}^{\prime}, \ldots, q_{n-1}^{\prime}, \xi_{0}\right)=g_{k}\left(q_{1}, \ldots, q_{n}, \xi_{0}\right)$ и $\Omega^{\prime}$ - любая ограниченная область изменения переменных $q_{j}^{\prime}, \xi_{0}, j=1, \ldots, n-1$, следовательно, величины $\left\|\partial g_{k}^{\prime} / \partial q_{s p}^{\prime}\right\|_{\Omega^{\prime}}$ равномерно ограниченны по $k$. Отсюда и из теорем вложения Соболева следует компактность $g_{k}^{\prime}$ в $\mathcal{L}_{2}\left(\Omega^{\prime}\right)$, а значит, и компактность $g_{k}$ в $\mathcal{L}_{2}(\Omega)$, что при учете $(3.9)$ приводит $\mathrm{K}(3.10)$.

3.8. Пусть $b(1)>a(1) \gg 1$ и вешественные функции $u_{1}(\tau), v_{1}(\tau)$ из $C^{2}\left(R^{(1)}\right)$ таковы, что $u_{1}(\tau)=1$ при $\tau \in[0 ; a(1)], 0<u_{1}(\tau)<1$ при $a(1)<\tau<b(1), u_{1}(\tau) \equiv 0$ при $\tau \geqslant$ $b(1), \quad u_{1}^{2}(\tau)+v_{1}^{2}(\tau)=1$. Положим $|q|_{0}^{2}=\sum_{j=1}^{n}\left|q_{j}\right|^{2} m_{j}, \quad \tau_{Z_{1}}=|q|_{0}, \quad u_{Z_{1}}=u_{1}\left(\tau_{Z_{1}}\right)$, $v_{Z_{1}}=v_{1}\left(\tau_{Z_{1}}\right)$. Пусть число $k$ велико и фиксировано, $\psi_{0, Z_{1}}=g_{k} u_{Z_{1}}, \hat{\psi}_{1}=g_{k} v_{Z_{1}}$. Тогда для каждого $\varepsilon>0$ сушествуют $a(1), b(1)$ и функция $u_{1}(\tau)$ такая, что для всех $k$

$$
\left(H g_{k}, g_{k}\right) \geqslant\left(H \psi_{0, Z_{1}}, \psi_{0, Z_{1}}\right)+\left(H \hat{\psi}_{1}, \hat{\psi}_{1}\right)-\varepsilon .
$$

Далее форму $\left(H \hat{\psi}_{1}, \hat{\psi}_{1}\right)$ оцениваем так же, как в работе [2] (см. с. 420-421), в результате чего получаем

$$
\left(H \hat{\psi}_{1}, \hat{\psi}_{1}\right) \geqslant \mu\left\|\hat{\psi}_{1}\right\|^{2}-\varepsilon
$$

Остается оценить величину $\left(H \psi_{0}, Z_{1}, \psi_{0}, Z_{1}\right)$. Это нельзя сделать аналогично работе [1], ибо в рассматриваемом нами случае область $\operatorname{supp} \psi_{0, Z_{1}}$ не ограничена. Пусть $\varphi_{k, b} \equiv$ $\psi_{0, Z_{1}}=g_{k} u_{Z_{1}}$. Покажем, что

$$
\lim _{k \rightarrow \infty}\left\|\varphi_{k, b}\right\|=0
$$

(тем самым мы исключаем второй случай п. 3.4). В силу (3.14)

$$
\left(H \varphi_{k, b}, \varphi_{k, b}\right) \geqslant c_{0}\left\|\varphi_{k, b}\right\|^{2}+\frac{1}{2}\left(T_{\mathrm{c} \perp} \varphi_{k, b}, \varphi_{k, b}\right) .
$$


Здесь и далее $c_{i}, i=0,1,2, \ldots,-$ константы, величины которых для нас не сушествен-

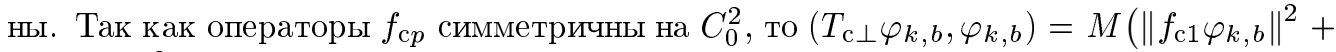
$\left.\left\|f_{\mathrm{c} 2} \varphi_{k, b}\right\|^{2}\right)$. Поэтому

$$
\left(T_{\mathrm{c} \perp} \varphi_{k, b}, \varphi_{k, b}\right)=(4 M)^{-1}\left\|\frac{d \varphi_{k, b}}{d \xi_{0}}\right\|^{2}+M^{-1} 4 B^{2} Q^{2}\left\|\xi_{0} \varphi_{k, b}\right\|^{2}+\left(\Phi \varphi_{k, b}, \varphi_{k, b}\right),
$$

где $\Phi$ есть линейная комбинация членов вида $q_{s p} q_{t p}, q_{s p} i d / d \xi_{0}$ и $q_{s p} \xi_{0}$. Поскольку при $\left(q, \xi_{0}\right) \in \operatorname{supp} \varphi_{k, b}$ выполняется неравенство $|q|_{0} \leqslant b(1)$, то

$$
\left|\left(\Phi \varphi_{k, b}, \varphi_{k, b}\right)\right| \leqslant \varepsilon\left\|\frac{d \varphi_{k, b}}{d \xi_{0}}\right\|^{2}+\varepsilon\left\|\xi_{0} \varphi_{k, b}\right\|^{2}+c_{1} \varepsilon^{-1}\left\|\varphi_{k, b}\right\|^{2},
$$

где $\varepsilon$ произвольно и $c_{1}$ зависит от $b(1)$. Фиксируем $\varepsilon<(4 M)^{-1}+2 B^{2} Q^{2} M^{-1}$. Тогда из (3.19)-(3.21) вытекает, что

$$
\left(H \varphi_{k, b}, \varphi_{k, b}\right) \geqslant Q^{2} B^{2} M^{-1}\left\|\xi_{0} \varphi_{k, b}\right\|^{2}-c_{3} .
$$

В силу (3.16), (3.17) и условия $\lim _{k \rightarrow \infty}\left(H g_{k}, g_{k}\right)=\nu$ выполняется $\sup _{k}\left(H \varphi_{k, b}, \varphi_{k, b}\right)<$ $+\infty$. Поэтому и вследствие (3.22) $K:=\overline{\lim }\left\|\xi_{0} \varphi_{k, b}\right\|<+\infty$. Но так как для любого $N>0 \lim _{k \rightarrow \infty}\left\|\xi_{0} \varphi_{k, b}\right\|_{\left|\xi_{0}\right| \leqslant N}^{2}=0$ согласно (3.10), то $\varlimsup\left\|\xi_{0} \varphi_{k, b}\right\|^{2} \geqslant N^{2} \varlimsup \lim _{\left\|\varphi_{k, b}\right\|^{2}}$ и, значит, $\varlimsup\left\|\varphi_{k, b}\right\| \leqslant K N^{-1}$ при любом $N>0$, т.е. $\varlimsup\left\|\varphi_{k, b}\right\|=0$, что доказывает (3.18). Следовательно, при больших $k$

$$
\left(H \varphi_{k, b}, \varphi_{k, b}\right) \geqslant \mu\left\|\varphi_{k, b}\right\|^{2}-\varepsilon .
$$

Отсюда и из $(3.16),(3.17)$ следует, что

$$
\underline{\lim }\left(H g_{v}, g_{k}\right) \geqslant \mu-\varepsilon .
$$

Так как значение $\varepsilon>0$ произвольно, то неравенство (3.11) можно считать доказанным.

3.9. Доказательство теоремы 2.3 проводится по той же схеме, что и доказательство теоремы 2.1. Пусть $a_{s p}, b_{0}, b_{1}$ - некоторые числа, $\varkappa=\sum_{j=1}^{n} \sum_{p=1}^{2} a_{j p} q_{j p}^{(1)}$. Проведем калибровочное преобразование

$$
e^{i \varkappa \lambda_{1}} H_{0}\left(Z_{2}, \lambda_{1}\right) e^{-i \varkappa \lambda_{1}},
$$

а затем перейдем в операторе (3.24) к новым переменным, полагая

$q_{s p}^{\prime}=q_{s p}^{(1)}, \quad s=1, \ldots, n, \quad p=1,2,3, \quad \lambda_{1}^{\prime}=\lambda_{1}, \quad \xi_{0}^{\prime}=\xi_{0}-b_{0} \lambda_{1}, \quad \xi_{1}^{\prime}=\xi_{1}-b_{1} \lambda_{1}$.

Обозначим полученный оператор через $H_{0}^{\prime}\left(Z_{2}, \lambda_{1}^{\prime}\right)$. Очевидно, что

$$
H_{0}^{\prime}\left(Z_{2}, \lambda_{1}^{\prime}\right)=\sum_{k=1}^{2}\left(T^{\prime}\left[C_{k}, \lambda_{k}^{\prime}\right]+V^{\prime}\left[C_{k}\right]\right)
$$


где

$$
\begin{gathered}
T^{\prime}\left[C_{k}, \lambda_{k}^{\prime}\right]=T_{\perp}^{\prime}\left[C_{k}, \lambda_{1}^{\prime}\right]+T_{03}^{\prime}\left[C_{k}\right], \\
T_{\perp}^{\prime}\left[C_{k}, \lambda_{1}^{\prime}\right]=\sum_{j \in C_{k}} \sum_{p=1}^{2} \frac{1}{m_{j}}\left[A_{j p}^{\prime}(k)+\sum_{\ell=0}^{1} h_{j p \ell}(k)+d_{j p}(k) \lambda_{1}^{\prime}\right], \\
d_{j p}(k)=\sum_{t \in C_{k}} a_{t p}\left(\delta_{j t}-\frac{m_{j}}{M_{k}}\right)-\frac{m_{j}}{2 M_{k}} \frac{M_{2}}{M}(-1)^{k}+F_{j}(k)\left(\frac{M_{1}}{M_{k}} b_{1}(-1)^{k}-b_{0}\right),
\end{gathered}
$$

a $A_{j p}^{\prime}(k), h_{j p \ell}^{\prime}, T_{03}^{\prime}\left[C_{k}\right]$ отличаются от $A_{j p}(k), h_{j p \ell}$ и $T_{03}\left[C_{k}\right]$ только штрихами у переменных. Подберем числа $a_{t p}, b_{0}$ и $b_{1}$ так, чтобы выполнялось равенство

$$
d_{j p}(k)=0, \quad j \in C_{k}, \quad k=1,2, \quad p=1,2 .
$$

Суммируя уравнения (3.25) по $j \in C_{k}$, получаем уравнения для $b_{0}$ и $b_{1}$ :

$$
b_{0}+b_{1}=M_{2}\left(4 M B Q_{1}\right)^{-1}, \quad b_{0}-b_{1} M_{1} M_{2}^{-1}=-M_{2}\left(4 M B Q_{2}\right)^{-1} .
$$

Найдя отсюда $b_{0}$ и $b_{1}$, подставим их в $(3.25)$, после чего отышем $a_{j p}$ в виде $a_{j p}=a(k) e_{j}+\tilde{a}(k) m_{j}$, где $a(k), \tilde{a}(k)$ - неизвестные константы. $\mathrm{B}$ результате получаем, что $a(k)=M_{2}(-1)^{k}\left(4 M Q_{k}\right)^{-1}$, а $\tilde{a}(k)$ может быть любым; мы возьмем $\tilde{a}(k)=0$. Таким образом, преобразование (3.24) и последуюшая замена переменных с найденными значениями $b_{0}, b_{1}, a_{j p}$ приводят к оператору $H_{0}^{\prime}\left(Z_{2}, \lambda_{1}^{\prime}\right)$ с $d_{j p}(k)=0$ при $j \in C_{k}, \quad k=1,2, \quad p=1,2$. Этот оператор, если отвлечься от штрихов у переменных, совпадает с $H_{0}\left(Z_{2}, 0\right)$. По построению $H_{0}\left(Z_{2}, \lambda_{1}\right), H_{0}^{\prime}\left(Z_{2}, \lambda_{1}^{\prime}\right)$ и $H_{0}\left(Z_{2}, 0\right)$ унитарно эквивалентны. Пусть $R_{0}^{(3 n-3)}=\left\{q^{(1)}, \xi_{0}, \xi_{1}, \lambda_{1}\right\}, R_{0}^{(3 n-4)}=\left\{q^{(1)}, \xi_{0}, \xi_{1}\right\}$. Поскольку оператор $H_{0}\left(Z_{2}, 0\right)$ не зависит от $\lambda_{1}$, то его спектры в $\mathcal{L}_{2}\left(R_{0}^{(3 n-3)}\right)$ и $\mathcal{L}_{2}\left(R_{0}^{(3 n-4)}\right)$ совпадают, но спектр в $\mathcal{L}_{2}\left(R_{0}^{(3 n-3)}\right)$ имеет дополнительное бесконечное вырождение по сравнению со спектром в $\mathcal{L}_{2}\left(R_{0}^{(3 n-4)}\right)$. Наконец, если рассматривать оператор $\widehat{H}_{0}\left(Z_{2}, \lambda_{1}\right)$ в $\mathcal{L}_{2}\left(R_{0}^{(3 n-4)}\right)$, то действуя аналогично предыдушему, но без замены $\lambda_{1}^{\prime}=\lambda_{1}$, мы убеждаемся, что оператор $\widehat{H}_{0}\left(Z_{2}, \lambda_{1}\right)$ унитарно эквивалентен $\widehat{H}_{0}\left(Z_{2}, 0\right)$. Теорема 2.3 доказана. Далее будем считать $Z_{2}$ фиксированным и положим $G=$ $\widehat{H}_{0}\left(Z_{2}, 0\right)$.

3.10. Доказательство теоремы 2.4 , как и доказательство теоремы 2.2 , состоит из двух частей. Сначала надо показать, что если $\nu \geqslant \mu\left(Z_{2}\right)$, то $\nu \in \sigma_{\mathrm{ess}}(G)$. Поскольку это делается аналогично п. 3.2, то мы опускаем соответствующие рассуждения. Далее необходимо доказать, что если $\nu \in \sigma_{\mathrm{ess}}(G)$, то

$$
\nu \geqslant \mu\left(Z_{2}\right)
$$

Так же, как в п. 3.2, 3.3, убеждаемся, что для доказательства (3.26) достаточно показать, что для любой последовательности $g_{m}=g_{m}\left(q^{(1)}, \xi_{0}, \xi_{1}\right)$ из $C_{0}^{2}$, для которой

$$
\sup _{m}\left(G g_{m}, g_{m}\right)<+\infty, \quad g_{m} \rightarrow 0 \quad \text { в } \quad \mathcal{L}_{2}\left(R_{0}^{(3 n-4)}\right), \quad\left\|g_{m}\right\|=1,
$$

выполняется неравенство

$$
\underline{\lim }\left(G g_{m}, g_{m}\right) \geqslant \mu\left(Z_{2}\right) .
$$


Пусть $\Omega_{b}=\left\{q^{(1)}, \xi_{0}, \xi_{1}|| q^{(1)} \mid \leqslant b\right\}$. Ключевую роль в доказательстве (3.28) играет равенство

$$
\lim _{m \rightarrow \infty}\left\|g_{m}\right\|_{\Omega_{b}}=0,
$$

которое мы сейчас докажем для любых $b>0$. Для получения (3.29) проведем перестройку кинетической части оператора $H_{0}\left(Z_{2}, 0\right)$, используя построения п. 3.5. Положим

$$
f_{j p}(k)=\left(A_{j p}^{(1)}(k)+\sum_{s=0}^{1} h_{j p s}(k)\right) m_{j}^{-1}, \quad p=1,2 ; \quad f_{j}(k)=\left(f_{j 1}(k), f_{j 2}(k)\right) .
$$

Тогда

$$
T_{\perp}\left[C_{k}\right]=\sum_{j \in C_{k}} \sum_{p=1}^{2} m_{j} f_{j p}^{2}(k)=T_{0 \perp}\left[C_{k}\right]+T_{\mathrm{c} \perp}\left[C_{k}\right]
$$

где

$$
\begin{gathered}
T_{0 \perp}\left[C_{k}\right]=\sum_{j \in C_{k}} \sum_{p=1}^{2}\left(f_{j p}(k)-f_{\mathrm{c} p}(k)\right)^{2} m_{j}, \quad T_{\mathrm{c} \perp}\left[C_{k}\right]=M_{k}\left(f_{\mathrm{c} 1}(k)^{2}+f_{\mathrm{c} 2}^{2}(k)\right), \\
f_{\mathrm{c} p}(k)=\sum_{g \in C_{k}} m_{j} f_{j p}(k) M_{k}^{-1}=\sum_{j \in C_{k}}\left(A_{j p}^{(1)}(k)+\sum_{\ell=0}^{1} h_{j p \ell}(k)\right) M_{k}^{-1} .
\end{gathered}
$$

Используя $(3.27)$ и симметричность операторов $f_{j p}(k)$ и $f_{\mathrm{c} p}(k)$, аналогично п. 3.7 получаем

$$
L:=\sup _{m, j, p}\left(\left\|\left(f_{j p}(k)-f_{c p}(k)\right) g_{m}\right\|^{2}+\left\|f_{c p}(k) g_{m}\right\|^{2}+\left\|B_{j 3}^{(1)}(k) g_{m}\right\|^{2}\right)<+\infty .
$$

Очевидно, что

$$
\begin{aligned}
\sum_{p, k=1}^{2} & \left\|f_{\mathrm{c} p}(k) g_{m}\right\|^{2} M_{k} \geqslant \sum_{p, k=1}^{2}\left\|f_{\mathrm{c} p}(k) g_{m}\right\|_{\Omega_{b}}^{2} M_{k}= \\
= & (4 M)^{-1}\left\|\frac{d g_{m}}{d \xi_{0}}\right\|_{\Omega_{b}}^{2}+\left(4 M M_{1}\right)^{-1} M_{2}\left\|\frac{d g_{m}}{d \xi_{1}}\right\|_{\Omega_{b}}^{2}+ \\
& +\sum_{k=1}^{2} 4 Q_{k}^{2} B^{2} M_{k}^{-1}\left\|\left(\xi_{1} M_{1} M_{k}^{-1}-(-1)^{k} \xi_{0}\right) g_{m}\right\|_{\Omega_{b}}^{2}+\left(\Phi_{1} g_{m}, g_{m}\right)_{\Omega_{b}},
\end{aligned}
$$

где $\Phi_{1}$ - линейная комбинация членов вида $q_{j p}^{(1)} q_{j^{\prime} p^{\prime}}^{(1)}, \quad\left(q_{j p}^{(1)} / i\right)\left(d / d \xi_{t}\right), q_{j p} \xi_{t}$. В силу (3.30), (3.31) сушествует $\delta_{0}>0$ такое, что

$$
L \geqslant \delta_{0}\left(\left\|\frac{d g_{m}}{d \xi_{0}}\right\|_{\Omega_{b}}^{2}+\left\|\frac{d g_{m}}{d \xi_{1}}\right\|_{\Omega_{b}}^{2}+\left\|\xi_{1} g_{m}\right\|_{\Omega_{b}}^{2}+\left\|\xi_{0} g_{m}\right\|_{\Omega_{b}}^{2}\right)-c
$$

при любых $b=b(1) \gg 1$; здесь $c=c(b)$. Пусть величина $N>0$ произвольна и

$$
\Omega_{b, N}=\left\{q^{(1)}, \xi_{0}, \xi_{1}|| q^{(1)} \mid \leqslant b(1), \xi_{0}^{2}+\xi_{1}^{2} \leqslant N^{2}\right\} .
$$


В силу (3.30), (3.31)

$$
\sup _{m, j}\left(\left\|B_{j p}^{(1)}(k) g_{m}\right\|_{\Omega_{b, N}}^{2}+\left\|\frac{d g_{m}}{d \xi_{0}}\right\|_{\Omega_{b, N}}^{2}+\left\|\frac{d g_{m}}{d \xi_{1}}\right\|_{\Omega_{b, N}}^{2}\right)<+\infty, \quad p, k=1,2, \quad j \in C_{k}
$$

и, значит, к последовательности $g_{m}$ в $\Omega_{b, N}$ применима теорема вложения Соболева. Используя ее и (3.27), получаем, что

$$
\lim _{m \rightarrow \infty}\left\|g_{m}\right\|_{\Omega_{b, N}}=0
$$

для любых значений $b$ и $N$. В силу $(3.32),(3.33)$ при фиксированном $b$ и любом $N$ выполняется неравенство $\varlimsup\|\|_{m} \|_{\Omega_{b}}^{2} \leqslant(L+c) /\left(\delta_{0} N\right)$, откуда и следует (3.29) при любых $b>0$.

3.11. Пусть числа $a(1), b(1)$ и функции $u_{1}(\tau), v_{1}(\tau)$ те же, что в п. $3.8, \tau_{C_{k}}^{2}=$ $\sum_{j \in C_{k}} m_{j}\left|q_{j}^{(1)}\right|^{2}, u_{C_{k}}=u_{1}\left(\tau_{C_{k}}\right), v_{C_{k}}=v_{1}\left(\tau_{C_{k}}\right)$. Положим $\psi_{0, C_{1}}=g_{m} u_{C_{1}}, \hat{\psi}_{1,1}=$ $g_{m} v_{C_{1}}, \psi_{0, Z_{2}}=\psi_{0, C_{1}} u_{C_{2}}, \hat{\psi}_{1,2}=\psi_{0, C_{1}} v_{C_{2}}$, где номер $m$ будем считать фиксированным. Тогда, выбирая при $\varepsilon>0$ числа $a(1)$ и $b(1)$ и функцию $u_{1}(\tau)$ аналогично $(3.16)$, получим

$$
\begin{aligned}
\left(G g_{m}, g_{m}\right) & \geqslant\left(G \psi_{0, C_{1}}, \psi_{0, C_{1}}\right)+\left(G \hat{\psi}_{1,1}, \hat{\psi}_{1,1}\right)-\varepsilon\left\|g_{m}\right\|^{2}, \\
\left(G \psi_{0, C_{1}}, \psi_{0, C_{1}}\right) & \geqslant\left(G \psi_{0, Z_{2}}, \psi_{0, Z_{2}}\right)+\left(G \hat{\psi}_{1,2}, \hat{\psi}_{1,2}\right)-\varepsilon\left\|\psi_{0, C_{1}}\right\|^{2} .
\end{aligned}
$$

Поскольку $\sum_{j \in C_{k}} q_{j}^{(1)} m_{j}=0$, а при $q^{(1)} \in \operatorname{supp} \hat{\psi}_{1, k}$ выполняется неравенство $\tau_{C_{k}}^{2} \geqslant$ $a(1)$, то оценка членов с $\hat{\psi}_{1, k}$ в (3.34) и (3.35) может быть проведена в точности так же, как оценка членов $\left(H \hat{\psi}_{1}, \hat{\psi}_{1}\right)$ в $(3.16)$, с той разницей, что интегралы по $R_{0}^{(3 n-4)}$ в скалярном произведении $\left(G \hat{\psi}_{1, k}, \hat{\psi}_{1, k}\right)$ надо записать как повторные: сначала по пространству $R_{0}\left[C_{k}\right]$ всех $q_{j}^{(1)}, j \in C_{k}, \sum_{j \in C_{k}} m_{j} q_{j}=0$, а потом по остальным переменным. Тогда, оценивая для больших $a(1)$ интегралы по $R_{0}\left[C_{k}\right]$ при фиксированных $\xi_{0}, \xi_{1}$ и $q_{j}^{(1)}$, $j \notin C_{k}$ аналогично (3.16) и (3.17), а потом интегрируя по фиксированным переменным, получим

$$
\left(G \hat{\psi}_{1, k}, \hat{\psi}_{1, k}\right) \geqslant \mu_{k}\left(Z_{2}\right)\left\|\hat{\psi}_{1, k}\right\|^{2}-\varepsilon\left\|\hat{\psi}_{1, k}\right\|^{2},
$$

где $\mu_{k}\left(Z_{2}\right)=\min _{Z_{3, k}^{\prime}} \inf G\left(Z_{3, k}^{\prime}\right)$ (см. п. 2.8) и через $Z_{3, k}^{\prime}=\left(C_{1}^{\prime}, C_{2}^{\prime}, C_{3}^{\prime}\right)$ обозначены те разбиения системы $Z_{1}$, которые получены из $Z_{2}=\left(C_{1}, C_{2}\right)$ дроблением кластера $C_{k}$. Очевидно, что $\mu_{k}\left(Z_{2}\right) \geqslant \mu\left(Z_{2}\right)$. Таким образом в (3.34) и (3.35) нам остается оценить только член с $\psi_{0}, Z_{2}$ По определению и в силу (3.29) при больших $m$

$$
\left(G \psi_{0, Z_{2}}, \psi_{0, Z_{2}}\right) \geqslant \mu\left\|\psi_{0, Z_{2}}\right\|^{2} \geqslant \mu\left(Z_{2}\right)\left\|\psi_{0, Z_{2}}\right\|^{2}-\varepsilon .
$$

Из (3.34)-(3.37) следует, что $\underline{\lim }\left(G g_{m}, g_{m}\right) \geqslant \mu\left(Z_{2}\right)-3 \varepsilon$; тем самым теорема 2.4 доказана.

\section{Список литературы}

[1] J. E. Avron, J. W. Herbst, B. Simon. Ann. Phys. 1978. V. 114. P. 431-451.

[2] С. А. Вугальтер, Г. М. Жислин. ТМФ. 1997. Т. 113. № 3. С. 413-431.

[3] Г. М. Жислин. ТМФ. 1999. Т. 118. № 1. С. 15-39; Т. 120. № 2. С. 291-308.

[4] Г. М. Жислин. Докл. РАН. 2001. Т. 377. № 3. С. 301-304.

[5] J. E. Avron, J. W. Herbst, B. Simon. Duke Math. J. 1978. V. 45. P. 874-883.

Поступила в редакцию 18.I.2002 г. 